\title{
Estimation of the Tire Contact Patch Length and Normal Load Using Intelligent Tires and Its Application in Small Ground Robot to Estimate the Tire-Road Friction
}

\begin{abstract}
REFERENCE: Khaleghian, S., Ghasemalizadeh, O., and Taheri, S., "Estimation of the Tire Contact Patch Length and Normal Load Using Intelligent Tires and Its Application in Small Ground Robot to Estimate the Tire-Road Friction," Tire Science and Technology, TSTCA, Vol. 44, No. 4, October-December 2016, pp. 248-261.
\end{abstract}

\begin{abstract}
Tire-road friction estimation is one of the most popular problems for the tire and vehicle industry. Accurate estimation of the tire-road friction leads to better performance of the traction and antilock braking system controllers, which reduces the number of accidents. Several researchers have worked in the field of friction estimation, and many tire models have been developed to predict the tire-road friction. In this article, an intelligent tire, which has an embedded accelerometer placed on the inner liner of the tire, is used to estimate the tire contact patch length parameter and normal load. To accomplish this, first, an existing tire testing trailer equipped with a force hub to measure tire forces and moments, a high-accuracy encoder to measure the angular velocity of the wheel, and VBOX, which is a global positioning systembased device, to estimate the longitudinal speed of the trailer was used. As a practical application for the normal load algorithm, a wheeled ground robot, which is equipped with several sensors, including an accelerometer and a flexible strain sensor inside the tire (used for terrain identification purposes), was designed and built. A set of algorithms was developed and used with the test data that were collected with both the trailer and the robot, and the contact patch length and the normal load were estimated. Also, the friction potential between the tire and the road was evaluated using a small ground robot.
\end{abstract}

KEY WORDS: intelligent tire, contact patch, normal load, friction, force estimation, neural network

\section{Introduction}

Routine measurement of normal force at tire-road contact patch has always been a challenging problem because of technical difficulties of mounting and implementing accurate sensors in tires. The intelligent tire concept has made it possible to measure various characteristics of tire-road interaction as well as estimate other parameters such as contact patch normal load.

Many studies have been done on integrating various types of sensors in tires $[1,2]$. The so called "intelligent tire" concept has been used specifically to

\footnotetext{
${ }^{1}$ Corresponding author. Department of Biomedical Engineering and Mechanics, Virginia Tech, Blacksburg, Virginia 24061, USA. Email: meysam@vt.edu

${ }^{2}$ Mechanical Engineering Department, Virginia Tech, Blacksburg, Virginia 24061, USA. Email: omidg@vt.edu

${ }^{3}$ Center for Tire Research (CenTiRe), Virginia Tech, Blacksburg, Virginia 24061, USA. Email: staheri@vt.edu
} 


\section{PATCH LENGTH AND NORMAL LOAD}

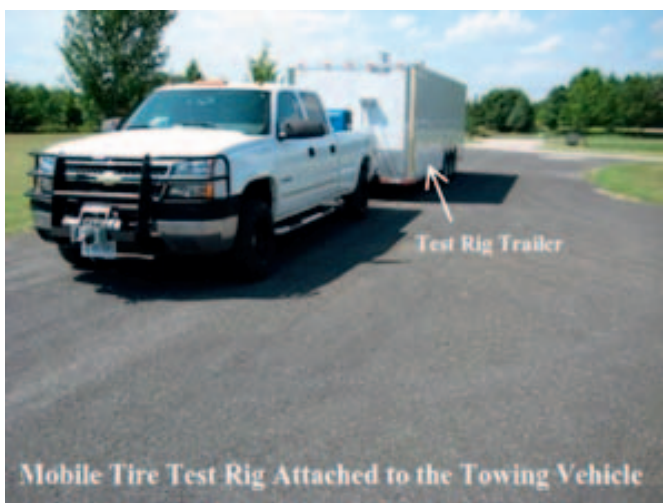

FIG. 1 - Trailer setup that is towed by a truck.

estimate tire-road friction in a few studies $[3,4]$ that shows the application of intelligent tires in terrain/ride characteristics identification [5,6]. On the other hand, estimation of tire forces has also been investigated in other works $[7,8]$. Although all of the estimations mentioned above help enhance vehicle/tire performance, estimation of contact patch length and normal load are the missing pieces of the puzzle. For instance, in suspension systems, engineers deal only with the normal loads including normal load at the contact patch. That information can be quite useful in designing active and semiactive suspensions where the tire load is considered as an unknown quantity in suspension systems analysis [9]. Even other tire studies will be able to use the normal force information such as studies in tire-terrain interaction $[10,11]$.

In this article, a simple approach is introduced to estimate the contact patch length and normal load on the tire with constant inflation pressure by training an artificial neural network. A parameter will be introduced that is proportional to the contact patch length. That parameter along with longitudinal velocity will be used as inputs to the neural network. Two sets of data will be used to train and validate the neural network, respectively. The same approach is also used in the small wheeled ground robot, and the dynamic equations of motion for the robot have been developed using a single-wheel vehicle dynamic model. A Kalman filter has been used to estimate longitudinal speed and tire-road friction coefficient.

\section{Test Setup}

A trailer test setup is used for this study that contains a quarter-car model, which is towed by a truck, as shown in Fig. 1 .

The quarter-car model setup has been equipped with various sensors to monitor the dynamics of the system. A six-degree-of-freedom force hub from Kistler is used to measure all the forces and moments in longitudinal, lateral, 

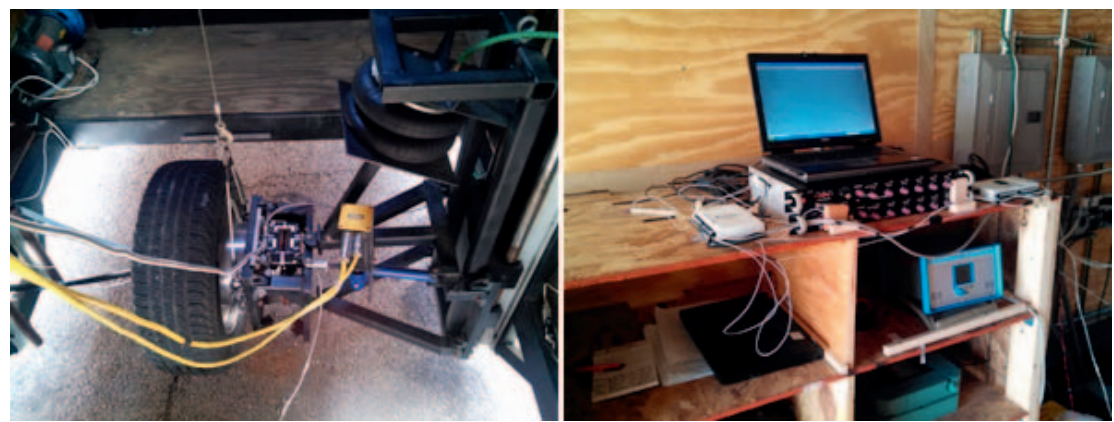

FIG. 2 - The quarter car setup and data-collecting and control system.

and vertical directions. An encoder is mounted to the force hub shaft to measure the angular speed of the wheel. The setup is shown in Fig. 2. VBOX, which is a global positioning system-based device, is used to measure the longitudinal speed of the trailer.

An air spring system is used to apply the desired normal load, which uses the force hub signal as feedback to control the normal force. Also a servo motor from Parker is used to apply the desired slip angle sweeps (steer angle). Both normal load and steering system are connected to a laptop through USB-NI DAQ and controlled by an algorithm written in LabView.

A triaxial accelerometer is mounted inside the tire to monitor the interaction between the tire and the road. This is shown in Fig. 3.

A data collection routine has been developed in LabView, which uses USBNI DAQ to synchronize and collect all the sensor data (acceleration signal from the sensor inside the tire, longitudinal speed of the trailer, angular speed of the wheel, and all the tire forces and moments). The sample rate that is used for this study was $1000 \mathrm{~Hz}$ for all the sensors, which is high enough for the purpose of
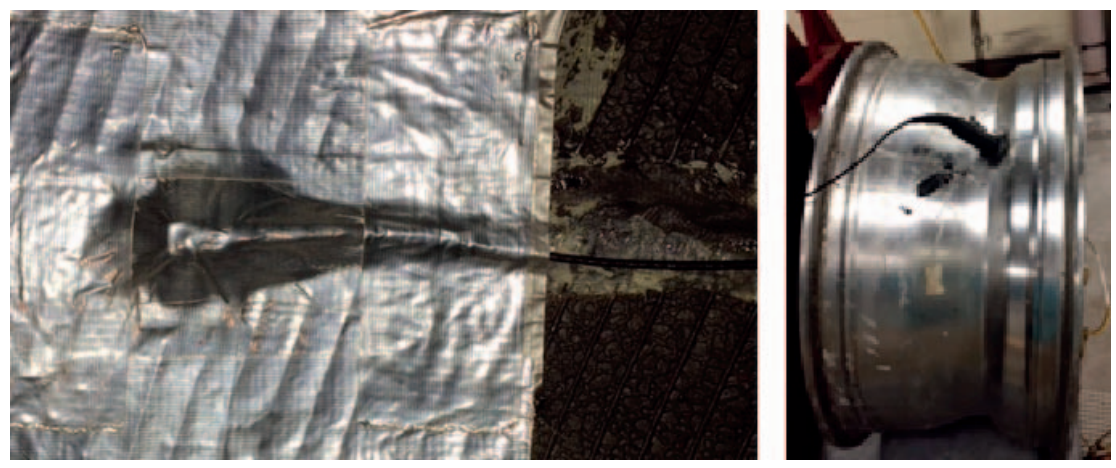

FIG. 3 - The accelerometer inside the tire. 


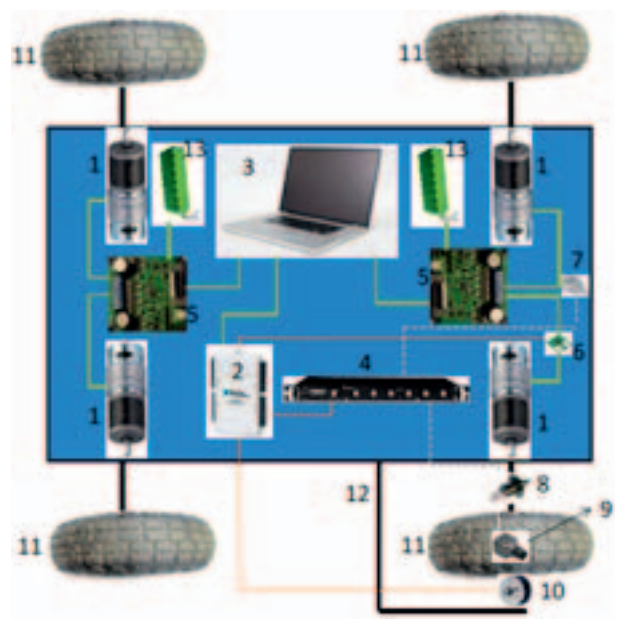

FIG. 4 - The schematic of the robot: 1: DC motor, 2: USB-NI DAQ, 3: laptop, 4: signal conditioner for the accelerometers, 5: motor controller, 6: current sensor, 7: single axis accelerometer, 8: slip ring, 9: triaxial accelerometer, 10: rotary encoder, 11: $0.248 \mathrm{~m}$ pneumatic tire, 12: mechanism to hold the encoder, 13: 24-V battery.

this study. For accurate estimation of contact patch length, a higher sample rate should be used to detect the exact edges of the contact patch; however, a very high sample rate is not practical, and a lower sample rate, like the one that was used for this study, still leads to an acceptable estimation of contact patch length.

In addition, a small wheeled ground robot was designed and built for this study; the chassis base was $0.361 \mathrm{~m}$ wide $\times 0.438 \mathrm{~m}$ long, which is placed on $0.254 \mathrm{~m}$ wheels (diameter). The schematic of the robot is shown in Fig. 4.

The robot has four 24-V brushed DC motors, which are controlled by two motor controllers. Each of the motor controllers has two channels to control two DC motors separately. One of the wheels is instrumented with a triaxial accelerometer; its angular velocity is measured using an accurate encoder, which is attached to the wheel shaft from the outside, as shown in the schematic picture (Fig. 4). A torque sensor is used to measure the input torque to the wheel from the motors (and uses the fact that in DC motors, the input current is proportional to the output torque). A single axis accelerometer is attached to the robot's chassis to measure the longitudinal acceleration. LabView is used to develop a data-collecting system for the robot, which also uses USB-NI DAQ. The overall weight of the robot with all equipment is about $29.5 \mathrm{~kg}$. A schematic view of the data-collecting system is shown in Fig. 5.

\section{Methodology}

Based on the literature, when the accelerometer enters and leaves the contact patch, two peaks are observed in a circumferential direction (as well as 


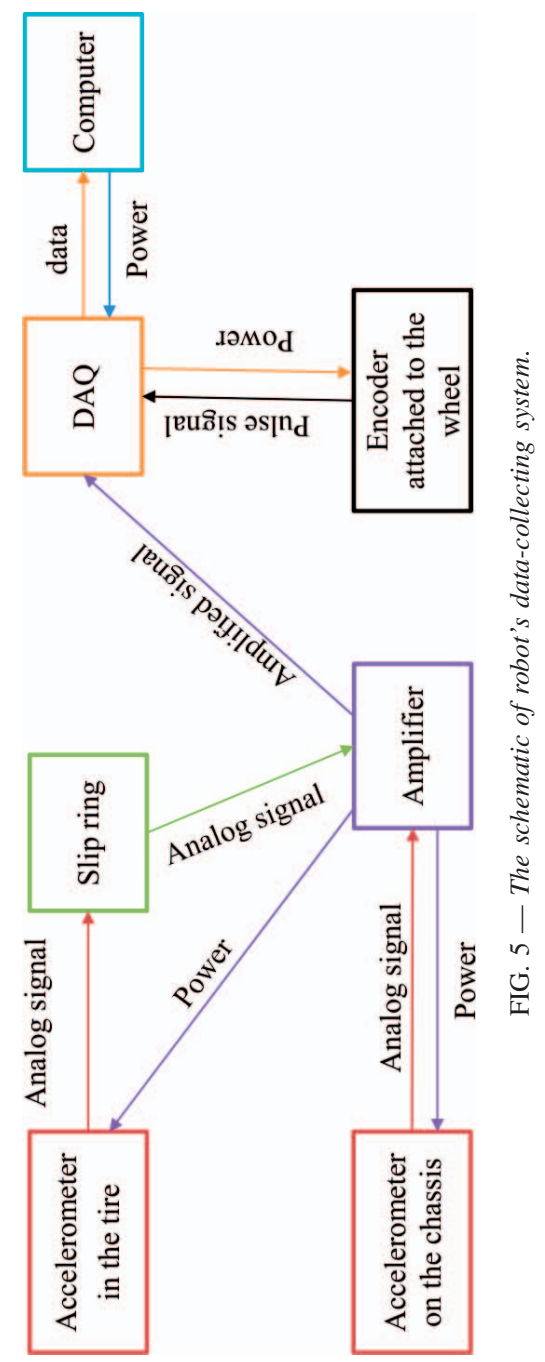




\section{PATCH LENGTH AND NORMAL LOAD}
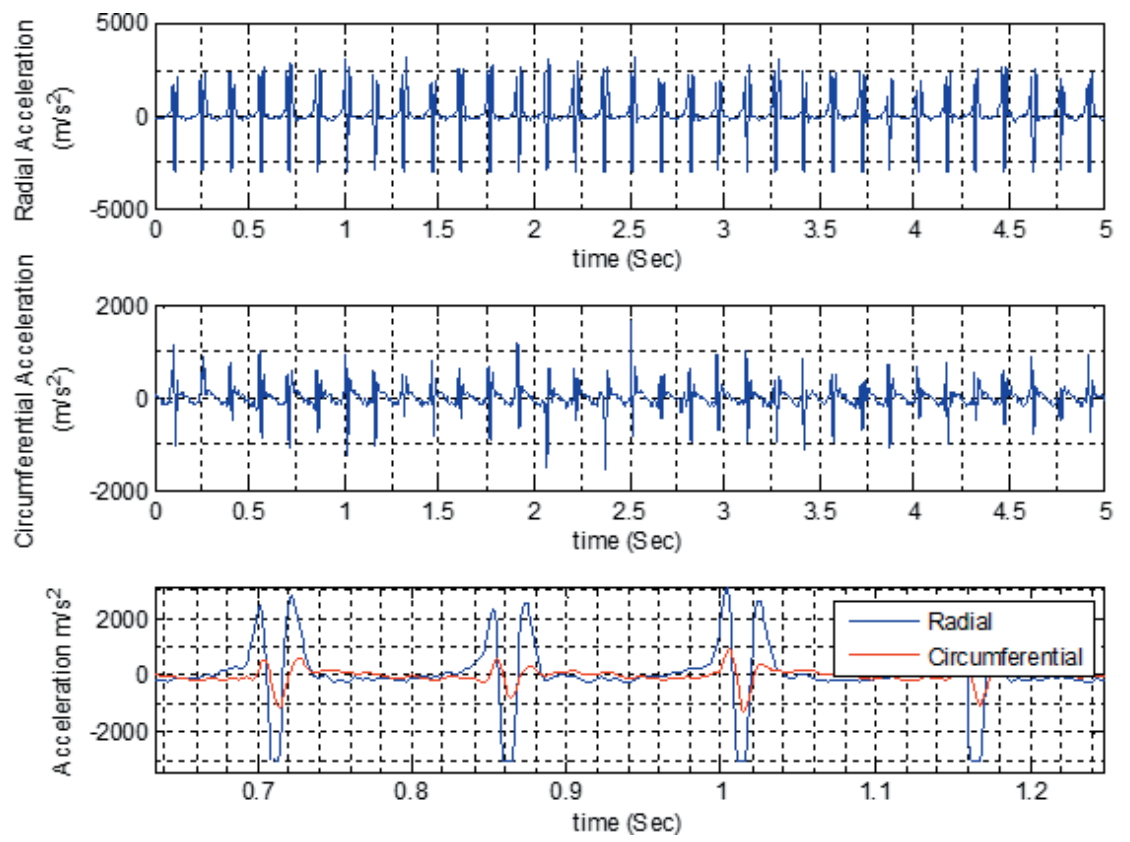

FIG. 6 - Radial and circumferential component of acceleration.

in a radial direction) of acceleration data, which represent the contact patch region [4,12]. Circumferential and radial components of the accelerometer are shown in Fig. 6 for a sample set of data.

A set of tests with three different normal loads and three different longitudinal speeds (shown in Table 1) is used to train a neural network to estimate the normal load using the trailer test setup. Each test has been repeated in both directions (the first test was performed in one direction and the second one in the opposite direction) to compensate for the effect of road grade, and the average data are used. Fig. 7 shows the algorithm that is used to estimate the normal load.

A computer code is developed that uses the encoder signal to extract the data for every tire revolution and detects the acceleration peaks. Once the time associated with each peak is detected, it should be multiplied by the trailer

TABLE $1-$ Different test conditions.

\begin{tabular}{lllll}
\hline & Trailer speed & & \multicolumn{3}{c}{ Normal load (N) } \\
\cline { 5 - 5 } mph & $\mathrm{km} / \mathrm{h}$ & & 3000 & 4000 \\
15 & 24.14 & 2000 & 3000 & 4000 \\
25 & 40.23 & 2000 & 3000 & 4000 \\
35 & 56.32 & 2000 & \\
\hline
\end{tabular}




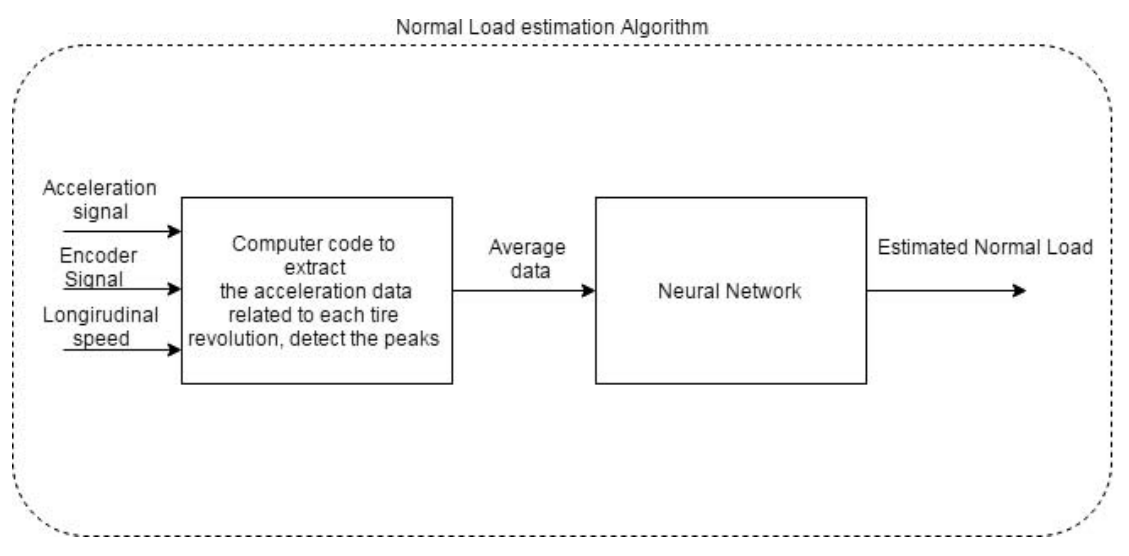

FIG. 7 - Normal force estimation algorithm.

velocity as follows:

$$
\text { contact patch length parameter }=\left(t_{\text {second peak }}-t_{\text {first peak }}\right) V_{\text {average }}
$$

A sample set of trailer data is depicted in Fig. 8. Because of the small fluctuation in normal load data, the average of normal load data for each test is used as the effective normal load.

The next step is to establish a relationship between the contact patch length parameter and the normal load. An artificial neural network (ANN)-based formulation was developed to estimate the tire normal load in terms of tire rolling speed, inflation pressure, and the contact patch length. A two-layer feedforward network with sigmoid hidden neurons and linear output neurons [13], which uses 10 neurons in its hidden layer and also is appropriate for a multidimensional mapping problem, is used to fit the data shown in Fig. 9.

The same approach is used for the robot to estimate the normal load. A Kalman filter has been used to determine the longitudinal velocity, angular velocity of the wheel, and friction force of the robot. A linear system can be modeled as a combination of measurements with a stochastic process [14]. The model is shown in Eqs. (2) and (3).

$$
\begin{aligned}
& x_{k}=A x_{k-1}+B u_{k}+w_{k} \\
& z_{k}=H_{k} x_{k}+v_{k}
\end{aligned}
$$

where $x_{k}$ is the state at time $k, u_{k}$ is the input control vector, $w_{k}$ is process noise, $z_{k}$ is the measurement made at time $k, v_{k}$ is additive measurement noise, and $H_{k}$ is the observation matrix. The process noise and measurement noise are assumed to be mutually independent random variables, spectrally white with 


\section{PATCH LENGTH AND NORMAL LOAD}
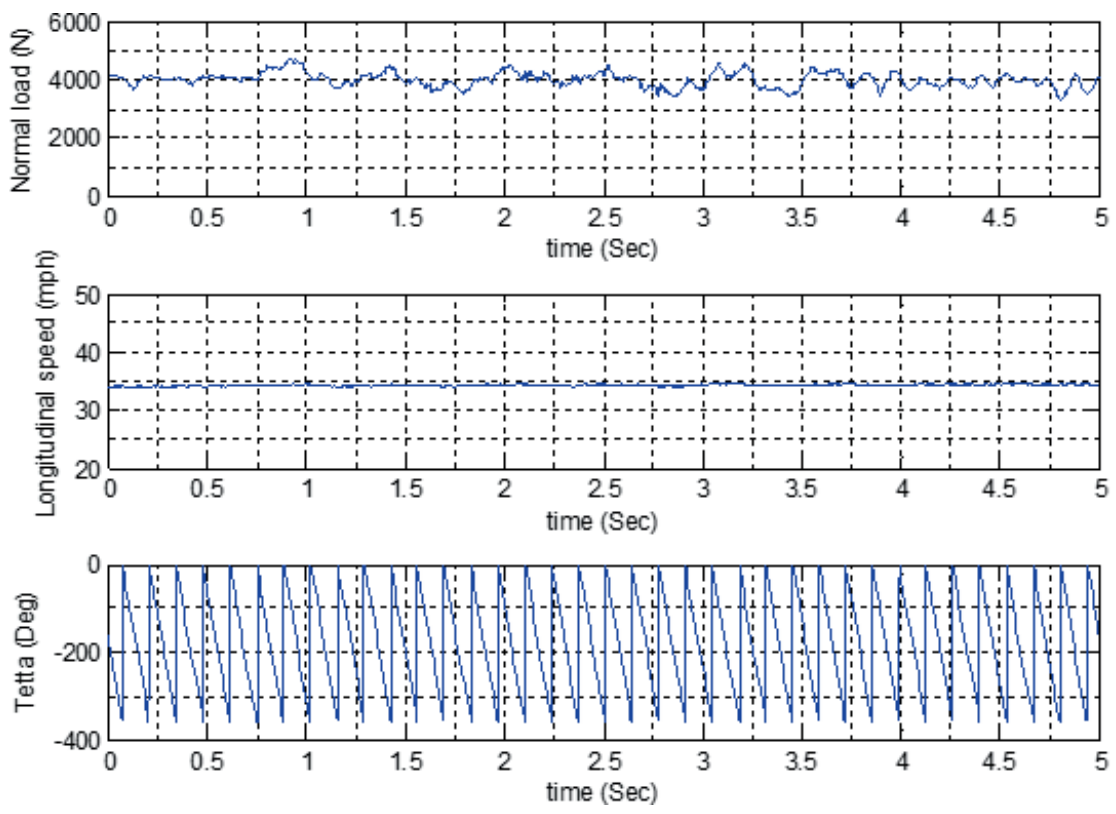

FIG. 8 - Normal force, longitudinal speed, and encoder signal (raw data).

normal probability distributions:

$$
\begin{gathered}
p(w)=\mathcal{N}(0, Q) \\
p(v)=\mathcal{N}(0, R)
\end{gathered}
$$

where process noise covariance $Q$ and measurement noise covariance $R$ matrices are assumed to be constant. The Kalman filter estimates the state by minimizing the posteriori estimation error covariance using two stages of prediction and correction.

$$
\text { prediction }\left\{\begin{array}{l}
\widehat{x}_{k}^{-}=A \widehat{x}_{k-1}+B u_{k} \\
\widehat{P}_{k}^{-}=A \widehat{P}_{k-1} A^{T}+Q
\end{array}\right.
$$

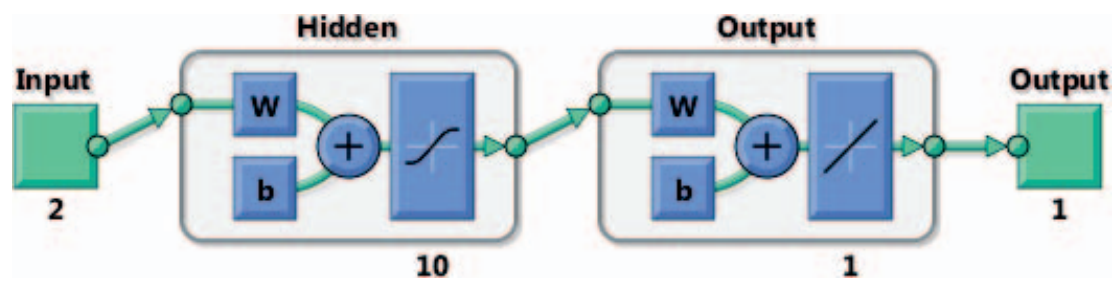

FIG. 9 - Two-layer feed-forward network, used to fit measured data. 

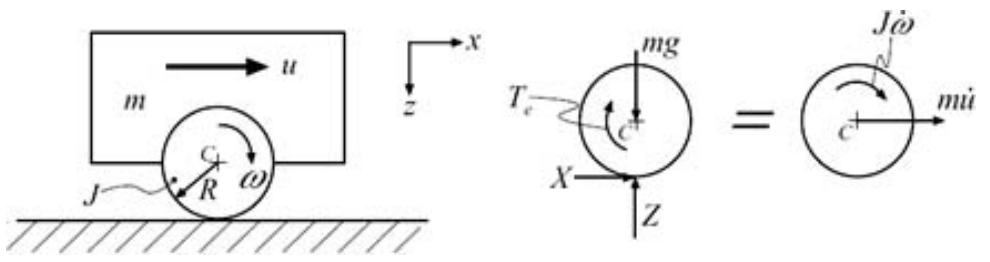

FIG. 10 - Free body diagram of single wheel model in acceleration mode [15].

$$
\text { correction }\left\{\begin{array}{l}
K_{k}=\widehat{P}_{k}^{-} H^{T}\left(H \widehat{P}_{k}^{-} H^{T}+R\right)-1 \\
\widehat{x}_{k}=\widehat{x}_{k}^{-}+K_{k}\left(z_{k}-H \widehat{x}_{k}^{-}\right) \\
\widehat{P}_{k}=\left(I-K_{k} H\right) \widehat{P}_{k}^{-}
\end{array}\right.
$$

where $K$ is the Kalman gain matrix and $I$ is the identity matrix. In this study, a state estimator is developed for computing the tire forces in addition to wheel and body speed for the friction estimation algorithm. The dynamics of the robot have been simplified using a single wheel dynamic model. This assumption is acceptable for this study since it is assumed that the robot can only go straight (there is no steer input). Also, since there is no suspension system, load transfer due to suspension deflection is assumed to be zero (no pitch dynamics). Fig. 10 shows the free body diagram of one wheel model in acceleration mode.

For the mathematical approximation, the longitudinal dynamics of the robot body are considered, and a random walk model has been assumed for the longitudinal friction force (according to the random walk model, it is assumed that $\dot{F}_{x}=\ddot{F}_{x}, \ddot{F}_{x}=0$ ). For braking mode, a linearized set of equations is given as follows:

$$
\begin{gathered}
\dot{u}=-\frac{1}{m_{b}} F_{x} \\
\dot{\omega}=-\frac{R_{w}}{J_{w}} F_{x}+\frac{1}{J_{w}} \tau \\
\dot{F}_{x}=\widehat{\dot{F}}_{x} \\
\ddot{F}_{x}=0
\end{gathered}
$$

where $u, \omega$, and $F_{x}$ are the body velocity, the individual wheel velocity, and the corresponding tire longitudinal force, respectively, $\tau$ is the summation of drive and brake torque, $m_{b}$ is one-fourth of the total mass of the robot, and $R_{w}$ and $J_{w}$ are the radius of the wheel and the second moment of inertia of the wheel, respectively. For this case $\mathrm{A}, \mathrm{B}$, and $\mathrm{H}$ matrices of Eqs. (2) and (3) are as follows: 


\section{PATCH LENGTH AND NORMAL LOAD}
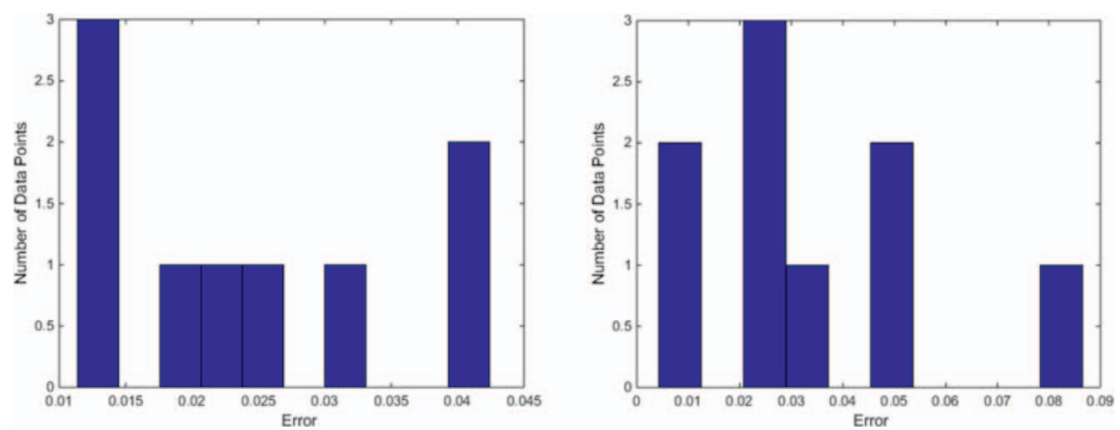

FIG. 11 - Left: Histogram of error percentages for the training data set. Right: Histogram of error percentages for the validation data set.

$$
A=\left[\begin{array}{cccc}
0 & 0 & -\frac{1}{m_{b}} & 0 \\
0 & 0 & -\frac{R_{w}}{J_{w}} & 0 \\
0 & 0 & 0 & 1 \\
0 & 0 & 0 & 0
\end{array}\right], B=\left[\begin{array}{c}
0 \\
\frac{1}{J_{w}} \\
0 \\
0
\end{array}\right], H=\left[\begin{array}{cccc}
0 & 0 & -\frac{1}{m_{b}} & 0 \\
0 & 1 & 0 & 0
\end{array}\right]
$$

\section{Results and Discussion}

For the normal load estimation algorithm with the trailer test setup, two sets of data were collected: one set was used to train the neural network using MATLAB Neural Network Toolbox, and the second data set was used to validate the trained neural network. The second set of data was taken with similar conditions to training data, but because of uncertainty in running the tests, the validation data points are not exactly equal to those of the training data.

The left graphic in Fig. 11 shows a histogram of the error percentages between estimated and measured normal forces for training data points, which indicates that the maximum percentage error of the trained data set is less than $4 \%$.

The right graphic in Fig. 11 shows the histogram of the error of the validation data points. As seen in the figure, the maximum percentage of error is less than $9 \%$. It should be noted that this set of data was not used in the training process of the neural network and was collected only for validation purpose.

It is normal to see larger error percentages with the validation data set compared with the training data set. It should be noted that only one data point of the validation set is close to $9 \%$ error, and the rest of the points are 


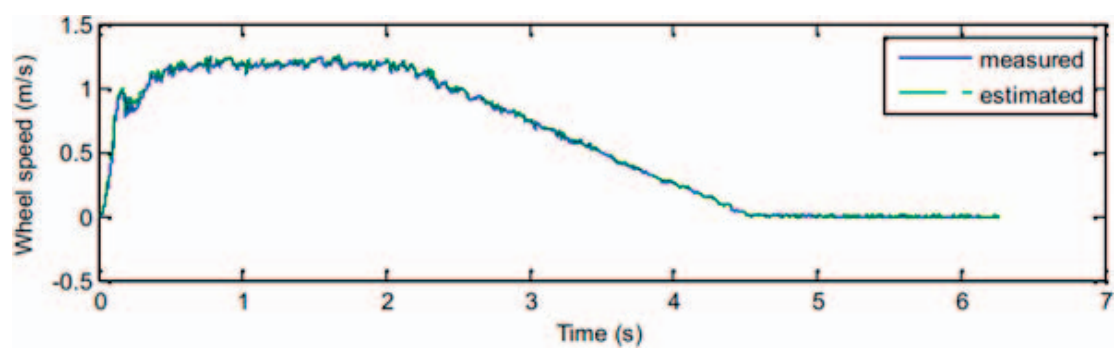

FIG. 12 - Measured and estimated wheel speed.

concentrated below 5\%. That point could be considered as an outlier. The same situation holds more or less with the training data set. Usually, one or two points have larger error percentages, but they are relatively far from the region where the rest of the points are concentrated.

An experiment was designed to validate the proposed estimation algorithm during a braking maneuver, and the small ground robot was used for this purpose. The robot starts from rest, and then an angular velocity of $95 \mathrm{rpm}$ $( \pm 5 \mathrm{rpm})$ is reached immediately and remains constant for 2 seconds. The brake is then applied gradually until the robot comes to a complete stop. The measured states are the angular velocity of the wheel and the longitudinal acceleration of the chassis. Also, the control signal is the input torque to the wheel from the DC motor, which is measured using the available torque sensor. The outputs of the estimation algorithm are the longitudinal speed, angular velocity of the wheel, and the friction force.

The estimated and measured wheel speeds and the estimated and measured longitudinal robot speeds are presented in Figs. 12 and 13, respectively. As observed in the figure, the estimation algorithm works properly, and the measured and estimated values of wheel speed (which is the radius $X$ the angular velocity of the wheel) and robot longitudinal speed are in good agreement.

According to Fig. 13, the maneuver can be divided into three different parts: the first part is accelerating, the second part is constant speed, and the third part is braking. The other output of the estimation algorithm is the friction force. Once the wheel speed, longitudinal speed, and the friction force is obtained using the Kalman filter, the friction coefficient and slippage value can be calculated as follows (the normal load was estimated using the same approach as with the intelligent tire):

$$
\begin{gathered}
\mu=\frac{F_{x}}{F_{N}} \\
\text { slip ratio }=\frac{R_{w} \omega-V}{V}
\end{gathered}
$$




\section{PATCH LENGTH AND NORMAL LOAD}

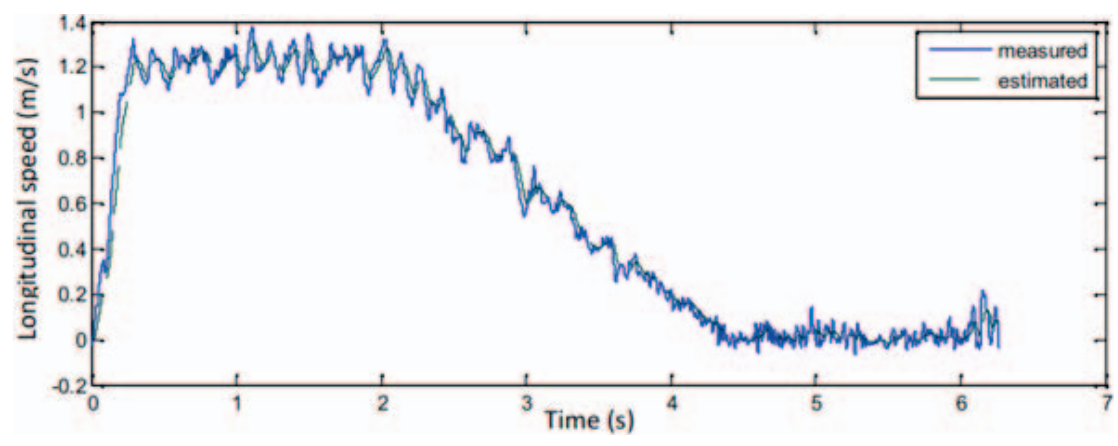

FIG. 13 - Measured and estimated longitudinal robot speed.

where $F_{N}$ is the normal force, $R_{w}$ is the radius of the wheel, and $V$ is the robot's longitudinal speed.

The friction coefficient versus slippage values for the braking part is plotted in Figure 14.

The saturated value of the $\mu$-slippage curve is in good agreement with the real value (rubber-wet asphalt), and the algorithm is validated.

\section{Conclusion}

Accurate estimation of tire normal load leads to better estimation of friction force and better performance of traction and antilock braking system controllers. In this study, a simple intelligent tire base algorithm is introduced to estimate the tire normal load. Although the sampling rate was not very high, it was sufficient to find a parameter related to contact patch length and accurate estimation of the normal load.

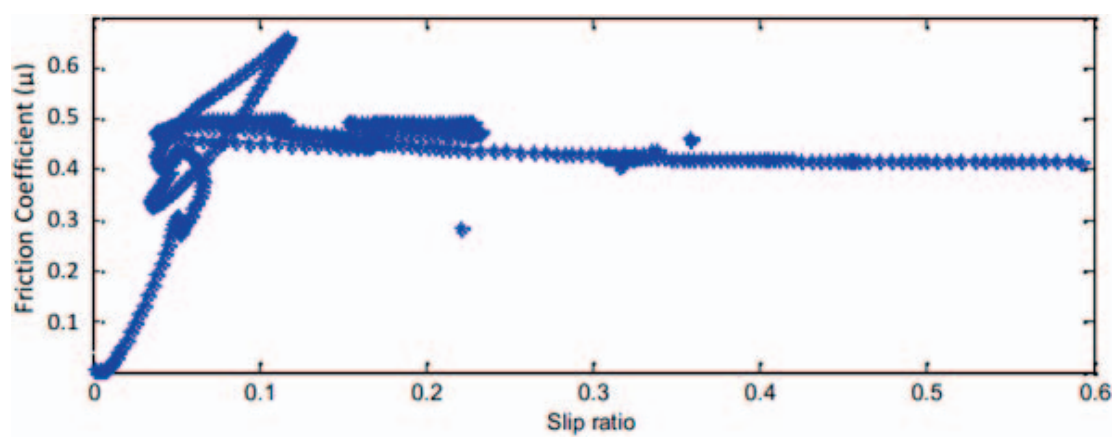

FIG. 14 - The friction coefficient for different slip. 
A set of tests with different applied normal loads was performed with the trailer test setup at different speeds, and a two-layer feed-forward network with 10 neurons in its hidden layers was developed to fit the processed data. To validate the algorithm, it was tested using another set of measured data, and good agreement was observed between measured and estimated normal loads. The proposed algorithm can be used easily in the vehicle industry to estimate the tire normal load; however, for each size of tire, a separate neural network should be trained and stored as a black box, which estimates the tire load.

As a practical application for the proposed algorithm, a similar approach is used for a small ground wheeled robot to estimate the normal load (a separate neural network was trained and used for this purpose) along with Kalman filterbased estimation algorithm to estimate the longitudinal velocity of the robot and the tire-road friction force. Finally, the estimated wheel speed, longitudinal robot speed, and friction coefficient were compared with the measured values; good agreement was observed, and the estimation algorithm was validated.

\section{References}

[1] Yilmazoglu, O., Brandt, M., Sigmund, J., Genc, E., and Hartnagel, H. L., "Integrated InAs/ GaSb 3D Magnetic Field Sensors for "the Intelligent Tire"," Sensors and Actuators A: Physical, Vol. 94, 2001, pp. 59-63.

[2] Zhang, X., Wang, F., Wang, Z., and He, D., "Intelligent Tires Based on Wireless Passive Surface Acoustic Wave Sensors," Proceedings. The 7th International IEEE Conference on Intelligent Transportation Systems, IEEE, 2004.

[3] Pohl, A., Reinhard S., and Reindl, L., "The "intelligent tire" Utilizing Passive SAW Sensors Measurement of Tire Friction," IEEE Transactions on Instrumentation and Measurement, Vol. 48, 1999, pp. 1041-1046.

[4] Singh, K. B., Arat, M. A., and Taheri, S., "An Intelligent Tire Based Tire-Road Friction Estimation Technique and Adaptive Wheel Slip Controller for Antilock Brake System," Journal of Dynamic Systems, Measurement, and Control, Vol. 135, 2013, p. 031002.

[5] Singh, K. B., Arat, M. A., and Taheri, S., "Development of a Smart Tire System and Its Use in Improving the Performance of a Collision Mitigation Braking System," ASME International Mechanical Engineering Congress and Exposition, Houston, TX, 2012, pp. 77-87.

[6] Arat, M. A., Singh, K. B., and Taheri, S., "An Intelligent Tire Based Adaptive Vehicle Stability Controller," International Journal of Vehicle Design, Vol. 65, 2014, pp. 118-143.

[7] Ray, L. R., "Nonlinear Tire Force Estimation and Road Friction Identification: Simulation and Experiments," Automatica, Vol. 33, 1997, pp. 1819-1833.

[8] Li, L., Wang, F.-Y., and Zhou, Q., "Integrated Longitudinal and Lateral Tire/Road Friction Modeling and Monitoring for Vehicle Motion Control," IEEE Transactions on Intelligent Transportation Systems, Vol. 7, 2006, pp. 1-19.

[9] Ghasemalizadeh, O., Taheri, S., Singh, A., and Goryca, J., "Semi-active Suspension Control Using Modern Methodology: Comprehensive Comparison Study," arXiv Preprint arXiv, 2014, 1411.3305 . 
[10] Taheri, S., Sandu, C., Taheri, S., Pinto, E., and Gorsich, D., "A Technical Survey on Terramechanics Models for Tire-Terrain Interaction Used in Modeling and Simulation of Wheeled Vehicles," Journal of Terramechanics, Vol. 57, 2015, pp. 1-22.

[11] Taheri, S., Sandu, C., and Taheri, S., "Development and Implementation of a Hybrid Soft Soil Tire Model (HSSTM)," Proceedings of the 18th International Conference of the ISTVS, Seoul, Korea, 2014.

[12] Matilainen, M. J., and Tuononen, A. J., "Intelligent Tire to Measure Contact Length in Dry Asphalt and Wet Concrete Conditions," International Symposium on Advanced Vehicle Control, Seoul, Korea, 2012.

[13] Duda, R., Hart, P., and Stork, D., Pattern Classification, 2nd ed., John Wiley \& Sons, New York, 2001.

[14] Doumiati, M., Charara, A., Victorino, A., and Lechner, D., Vehicle Dynamics Estimation Using Kalman Filtering: Experimental Validation, Wiley-ISTE, New York, 2012.

[15] Olson, B. J., Shaw, S. W., and Stepan, G., "Nonlinear Dynamics of Vehicle Traction," Vehicle System Dynamics, Vol. 40, 2003, pp. 377-399. 